\title{
A Method for Automatic 3D Measurement of Unknown Objects Based on the Structured Light System
}

\author{
Qiang Zhao, Bingwei He, Liwei Zhang and Shengsheng Dong \\ The School of Mechanical Engineering and Automation, Fuzhou University, PR China
}

\begin{abstract}
This paper proposes a novel approach to determine three-dimensional (3-D) model from a minimum number of viewpoint of an object. Calculating the position relation location of two coordinate system in order to splice the multiview point cloud. The Next Best Views (NBV) is calculated by the visibility classification and Mean-shift algorithm and then the overall morphology of the measured object can be acquired. The view planning method not only reaches a fast measurement speed, but also possesses a high fidelity to the complex regions of the measured object by the experiments.
\end{abstract}

Keywords-coordinate transformation; next best view; 3-D measurement; view planning

\section{INTRODUCTION}

Automatically constructing 3-D models of objects are widely used in many applications such as entertainment, industrial and the protection of cultural heritage, as well as architecture. The 3-D coordinate data was acquired from the visual system, 3-D model of scenes and objects was reconstructed to confirm shape, size position of the object in 3-D space.

Many researchers has put forward a variety of NBV argues algorithms. Goesele et al. [1] and Gallup et al. [2] select viewpoints relying on simple properties of the input images such as resolution or baseline. Furukawa et al. [3] divide the camera set into clusters to make the multi-view stereo scalable, and also remove redundant images. Content-aware approaches use additional cues to consider the appearance and the geometric properties of the model. The selection algorithm of Hornung et al. [4] relies on coverage and visibility cues to guarantee a minimum reconstruction quality and then refines the most difficult regions using photo-consistency. Tarbox and Gottschlich's seminal work [5] introduced a number of important concepts, including the idea of a measurability matrix capturing a complete visibility analysis over the set of all surface points and all admissible viewpoints. Zha et al. [6] similarly represented the unscanned areas of the viewing volume with vectors "attached" to the boundaries of surface meshes. Several methods targeted at reconstruction have been put forward since (e.g., by Larsson and Kjellander [7] and by Scott himself [8]) these attempt to solve the next best view problem for unknown object surface reconstruction, but that do not fit structured light system. Maver et al. [9] proposed solutions using max-min principle from all possible spatial position to determine the next best view. However, this method could only be used for specific configuration of a visual sensor system. Yuan [10] put forward a method of group-based vector-chain for view planning. But this method could only get the next viewpoint of directional parameters. Loriot [11] established rapid methods for view planning to ensure maximum coverage of the object's surface, but this method is very expensive in robot's movements.

The rest of this paper is organized as follows. In section II, it introduces the coordinate transformation and stitches the multi-view point cloud. In section III, the angle criterion and the shielding criterion for viewpoint planning is introduced. In section IV, the NBV is determined. In section V, experimental results are given by implementing the proposed method, followed by conclusions and future lines in section VI.

\section{COORDINATE TRANSFORMATION}

\section{A. Calibration with Double Ball}

Measurement system transforms the different viewpoints of the object by lateral rotation and overturn of two axis turntable. Therefore, it necessary to calibrate the relationship of two axis turntable and coordinate system, and taking advantage of the result to stitch the multi-view point cloud.

The pose of rotation axis and roll axis in the projector coordinate system are calculated. Shown in Fig.1 (a), two axes turntable will roll different locations, and the 3-D measurement system is used to measure the position of the calibration ball, and gets two goals' point clouds in the projector system. Respectively fitting two balls of twelve spatial location to circle, to give the two calibration ball roll center $A\left(x_{1}, y_{1}, z_{1}\right)$ and $B\left(x_{2}, y_{2}, z_{2}\right)$. So the line $A B$ is the roll axis of two axis turntable. Similarly, as shown in Fig.1 (b), the two calibration ball rotation center $C\left(x_{3}, y_{3}, z_{3}\right)$ and $D\left(x_{3}, y_{3}, z_{3}\right)$ can be obtained. Line CD is the rotation axis of two axis turntable.
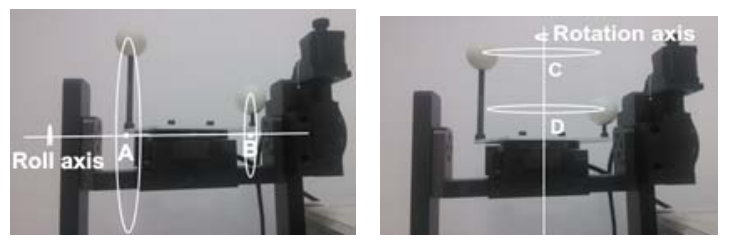

FIGURE I. (a) CALIBRATE THE POSITION OF ROLL AXIS IN THE PROJECTOR COORDINATE SYSTEM; (b) CALIBRATE THE POSITION OF ROTATION AXIS IN THE PROJECTOR COORDINATE SYSTEM 


\section{B. Calculate the Position Relation}

According to the equation of line $\mathrm{AB}$ and line $\mathrm{CD}$, solving the point $\mathrm{O}$ that is the intersection of two straight lines by the least squares method. Remember the line $\mathrm{AB}$ and CD direction the vector are $\vec{n}_{1}$ and $\vec{n}_{2}$, with $\mathrm{O}$ as origin in the two axis turntable system, and the vector $\vec{n}_{1}, \vec{n}_{2}$ as $\mathrm{x}$ and $\mathrm{z}$ axes, to establish a fixed turret coordinates, as shown in Fig. 2.

According to the three-point alignment transform method, the transformation of the relationship between the projector coordinate system and the fixed platform coordinates can be calculated as follow:

$$
X=\left(X_{p} R_{p}+T_{p}\right)^{T}
$$

where $R_{p}$ and $T$ are the rotation matrix and translation matrix, $X_{p}$ and $X$ are the spatial points in projectors coordinates and in fixed platform coordinate system.
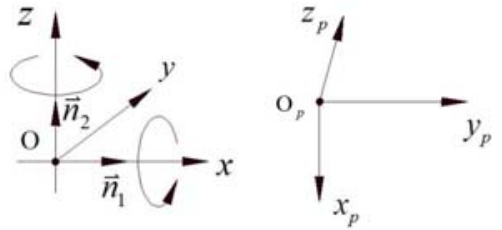

FIGURE II. FIXED PLATFORM COORDINATE X AND PROJECTOR COORDINATE $\mathrm{X}_{\mathrm{p}}$

\section{Matching}

As shown in Fig.3, the object point $\mathrm{Q}$ in the fixed platform coordinate rotates $\theta$ around the $\mathrm{z}$ axis (transformation 1) to obtain the point $Q^{\prime}$, and then roll $\alpha$ around the $\mathrm{x}$ axis (transformation 2) to obtain the points $Q^{\prime \prime}$. Therefore, the obtained object points are rotated $\theta$ around the $\mathrm{z}$ axis and rolled $\alpha$ around the $\mathrm{x}$ axis by the measurement system and inverted object point coordinates in the projector coordinate.

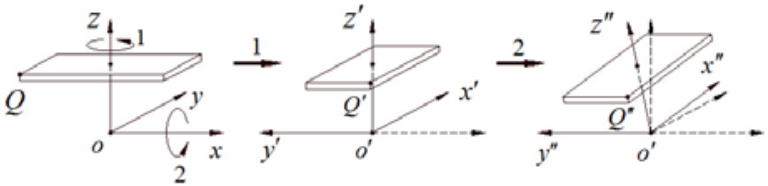

FIGURE III. THE COORDINATE TRANSFER SCHEMATIC OF POINT Q

To achieve multi-view point cloud splicing, the measured point will be transformed from the projector coordinate to the fixed platform coordinate $\mathrm{X}$, and then rotate and roll transformation, get the measured point coordinate in the fixed platform coordinates. The transformation formula of rotating around $\mathrm{x}$-axis and rolling around $\mathrm{z}$-axis are shown (2) and (3) as follows, in respectively:

$$
\begin{gathered}
X_{Q^{\prime}}=\left(\begin{array}{ccc}
\cos \theta & -\sin \theta & 0 \\
\sin \theta & \cos \theta & 0 \\
0 & 0 & 1
\end{array}\right) X_{Q}=R_{\theta} X_{Q} \\
X_{Q^{\prime \prime}}=\left(\begin{array}{ccc}
1 & 0 & 0 \\
0 & \cos \alpha & -\sin \alpha \\
0 & \sin \alpha & \cos \alpha
\end{array}\right) X_{Q^{\prime}}=R_{\alpha} X_{Q^{\prime}}
\end{gathered}
$$

Comprehensive formula (1), (2) and (3), transformational matrix can be solved, which the point in projector coordinate system is transformed to point in fixed platform coordinate system:

$$
X_{Q}=R_{\theta}^{-1} X_{Q^{\prime}}=R_{\theta}^{-1} R_{\alpha}^{-1} X_{Q^{\prime \prime}}=R_{\theta}^{-1} R_{\alpha}^{-1}\left(X_{p Q^{\prime \prime}} R_{p}+T_{p}\right)
$$

Therefore, the point cloud of different viewpoints can be spliced in accordance with the formula (4). The calibration process has still a certain error, so it needs to carry on the ICP [12] accuracy of registration based on the results of this registration.

\section{THE CRITERION FOR VIEW PLANNING}

\section{A. The Angle Criterion}

Souhaiel Khalfaoui proposed an efficient method for fully automatic 3D digitization of unknown objects [13]. In this method, it can preliminarily judge visibility of the 3-D point with respect to the given viewpoint, by calculating the angle between the viewing direction and normal vector of 3$\mathrm{D}$ point. As shown in Fig. 6, the green part is the WV point, and the red part is the BV points. Angle criterion can be expressed as:

$$
V_{a}^{i}\left(f_{j}\right)=\left\{\begin{array}{l}
1,0<\alpha_{j}^{i}<\pi / 3 \\
0, \text { others }
\end{array}\right.
$$

where, $\alpha_{j}^{i}$ is the angle between the normal direction of the point $\mathrm{j}$ and the $\mathrm{i}$ viewing direction, $V_{a}^{i}\left(f_{j}\right)$ is the result that the visibility of point $\mathrm{j}$ with respect to the viewpoint $\mathrm{i}$ from the angle criterion: if $V_{a}^{i}\left(f_{j}\right)=1$ represent that is $\mathrm{WB}$; otherwise, that is BV.

From the perspective of the criterion of the first viewpoint point cloud object classification, the result shown in Fig.4 (a). By the angle criterion to classify the first point of the object point cloud, the result is shown in Fig.4 (b), the green part is the WV points, and the red part is the BV points. 


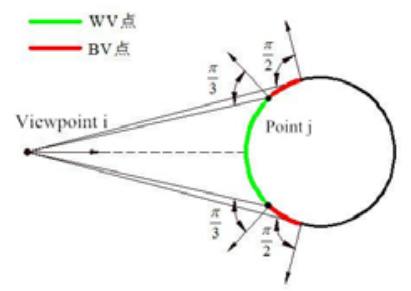

(a)

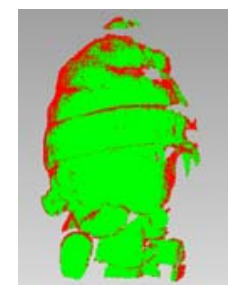

(b)
FIGURE IV. (a) THE CLASSIFICATION RESULTS OF THE SURFACE POINTS OF THE OBJECT BY THE ANGLE CRITERION; (b) THE CLASSIFICATION RESULTS OF A VIEW POINT CLOUD BY THE ANGLE CRITERION

\section{B. The Shielding Criterion}

In the process of reconstruction, objects may be blocked due to their own shapes. Therefore, it can't determine point whether is WB, only depending on the angle criterion. Shown in Fig.5 (a), the contour AB of objects with respect to viewpoint 2 is $\mathrm{WB}$ by the angle criterion, but the $\mathrm{AB}$ is blocked by the viewpoint 1 . Therefore, it needs to introduce the shielding criterion to improve the classification of the 3D surface points. The principle of shielding criterion is ray tracing. Tracking in the opposite direction, along arriving the light of viewpoint $i$, getting rays that the surface points to get ray image point. The point with respect to the viewpoint $i$ is WB if the ray is not blocked by object, otherwise, it is BV. Therefore, the shielding criterion can be summarized as follows:

$$
V_{r}^{i}\left(f_{j}\right)= \begin{cases}1, & \text { Ray tracing is not blocked } \\ 0, & \text { Ray tracing is blocked }\end{cases}
$$

where, $V_{r}^{i}\left(f_{j}\right)$ is the result that the visibility of point $\mathrm{j}$ with respect to the viewpoint $\mathrm{i}$ from the shielding criterion: if $V_{r}^{i}\left(f_{j}\right)=1$ represent that is $\mathrm{WB}$; otherwise, that is $\mathrm{BV}$.

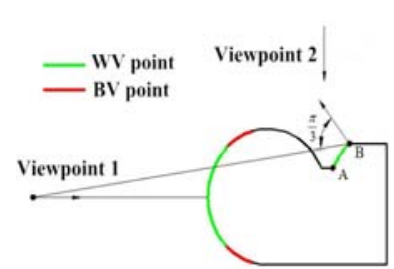

(a)

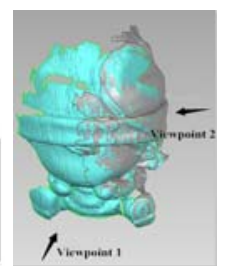

(b)

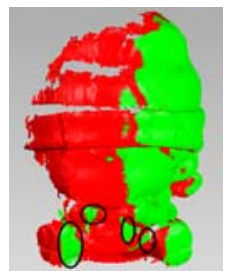

(c)
FIGURE V. (a) THE FAILURE CASE OF THE SHIELDING CRITERION; (b) THE LOCATION RELATIONSHIP OF TWO VIEWPOINTS CLOUDS; (c) THE VISIBILITY

CLASSIFICATION OF TWO VIEWPOINTS CLOUDS WITH RESPECT TO VIEWPOINT 2

In the process of object measuring, it also can appear blocking problem. According to the Angle criterion, as shown in Fig.5 (c), the circle mark in several point cloud with respect to the viewpoint 2 is WB but as shown the Fig.5 (b), there is blocked for these point clouds with respect to the viewpoint 2 . Therefore, it needs to add the shielding criterion to improve the accuracy of classification of visibility for the points cloud.
In order to improve the efficiency of data classification, the shielding criterion only used to analyze WV points obtained by the angle criterion, if that WV points is blocked, it will be reclassified to $\mathrm{BV}$ points. It can be classified for the viewpoint $1,2,3 \ldots$ i clouds, which considerate the two criterion. TABLET I, it analyzes the calculation of BV points by $\mathrm{i}=2$, where $\mathrm{N}$ is number of the total points in the two viewpoints. $\mathrm{j}=1,2,3 \ldots \mathrm{N}, V_{a}^{i}\left(f_{j}\right)$ and $V_{r}^{i}\left(f_{j}\right)$ are the results of visibility about point $j$ with respect to the viewpoint $i(i=1,2)$ by the angle criterion and the shielding criterion.

TABLE I. DETERMINE THE BV POINTS FROM THE FIRST VIEWPOINT AND THE SECOND VIEWPOINT POINT CLOUD

\begin{tabular}{c|c|c|c|c|c|c|c|c|c|c|c|c}
\hline Visibility & \multicolumn{10}{|c}{$j$} \\
\cline { 2 - 14 } & 1 & 2 & 3 & 4 & 5 & 6 &.. &.. &.. &.. &.. & $N$ \\
\hline$V_{a}^{1}\left(f_{j}\right)$ & 0 & 1 & 0 & 1 & 0 & 0 & 0 & 1 & 0 & 1 & 0 & 1 \\
\hline$V_{r}^{1}\left(f_{j}\right)$ & - & 1 & - & 0 & - & - & - & 1 & - & 1 & - & 1 \\
\hline$W V^{1}$ and $B V^{1}$ & 0 & 1 & 0 & 0 & 0 & 0 & 0 & 1 & 0 & 1 & 0 & 1 \\
\hline$V_{a}^{2}\left(f_{j}\right)$ & 1 & 0 & 0 & 0 & 1 & 1 & 0 & 1 & 1 & 1 & 0 & 1 \\
\hline$V_{r}^{2}\left(f_{j}\right)$ & 0 & - & - & - & 0 & 1 & - & 1 & 0 & 1 & - & 1 \\
\hline$W V^{2}$ and $B V^{2}$ & 0 & 0 & 0 & 0 & 0 & 1 & 0 & 1 & 0 & 1 & 0 & 1 \\
\hline$B V^{1} \cap B V^{2}$ & 0 & - & 0 & 0 & 0 & - & 0 & - & 0 & - & 0 & - \\
\hline
\end{tabular}

As shown in Tablet I, each of point is traversed by the angle criterion, but the shielding criterion for the being classified as BV points. Because of the significance of the NBV is that the largest number of BV points for optimal measurement, so it is necessary to solve the first viewpoint and the second viewpoint are $\mathrm{BV}$ points that is assembly $E^{2}=B V^{1} \cap B V^{2}$. For the general case, assembly

$$
E^{i}=\bigcap_{k=1}^{i} B V^{k}
$$

Assembly $E^{i}$ is consisted of the BV points with the currently measured point cloud for all viewpoints before $i$, the direction of viewpoint $\mathrm{i}+1$ will be calculated by these point clouds. For point $\mathrm{j}$, the point before $\mathrm{i}$ viewpoints whether were $\mathrm{BV}$ is determined by the following function:

$$
F\left(n_{j}\right)=\max \left\{\min _{p=1: i}\left\{V_{a}^{p}\left(f_{j}\right), V_{r}^{p}\left(f_{j}\right)\right\}\right\}
$$

where, the $p$ is the sequence value to the viewpoint, $p=1,2$, $3 \ldots i$. If and only if point $\mathrm{j}$ with respect to before $\mathrm{i}$ viewpoints are $\mathrm{BV}, F\left(n_{j}\right)=0$, the rest of the situation, $F\left(n_{j}\right)=1$. So, the NBV will be obtained by the analysis BV points of all the before viewpoint $i$.

\section{DETERMINING THE NBV}

\section{A. Mean Shift Application}

The points of all viewpoints before $\mathrm{i}$ can be classified by the criterion, so all the points will be classified to BV points or WV points. The spherical space can be constituted by 
using the outside normal of BV points. BV point $j$ correspond spherical point $S_{j}$ is the best observation position. With this transformation of thinking to determine the viewpoint with the dense BV points, and use it as the NBV of the alternative viewpoints. As shown in Fig.6 (a), the outside normal of BV points in objects are extended to obtain the spherical discrete points contained the boundary information of object. It can be performed by the clustering analysis, and the result is shown in Fig.6 (b). It shows that the center of the cluster by cluster analysis does not coincide with that centroid of the obtained cluster. The several locations with more focused of sphere point as the NBV alternative viewpoint.

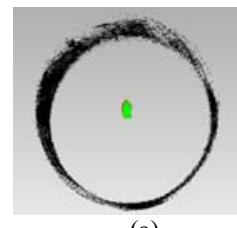

(a)

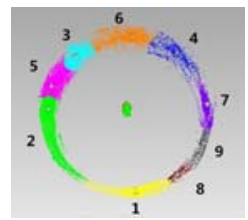

(b)
FIGURE VI. (a) CALCULATE THE SPHERICAL SPACE WITH BV POINTS; (b) THE RESULT OF CLUSTER ANALYSIS WITH SPHERE POINTS

\section{B. Evaluation of Alternative Viewpoint}

For the evaluation of alternative viewpoints, the evaluation function is given for selecting the NBV, the number of $\mathrm{BV}$ points can be observed by the alternative viewpoint and the distance of path between the viewpoint $i$ and the alternative viewpoint are quantified the weight of the two factors in the evaluation function. Thus, the evaluation function will be given as follows:

$$
g_{i}(m)=U_{N} \frac{N_{m}}{\sum_{k=1}^{M} N_{k}}-U_{L} \frac{L_{m}}{\sum_{k=1}^{M} L_{k}}, m=1,2,3, \ldots, M
$$

where the $g_{i}(m)$ is evaluation function values for the $\mathrm{m}$ alternative viewpoints in the viewpoint $\mathrm{i}+1 . U_{N}$ and $U_{L}$ are respectively the weights of points and distance of the alternatives. $N_{m}$ is the number of spherical points for an alternative viewpoint $\mathrm{m}, L_{m}$ is the sum of the required angle of rotation and roll from the viewpoint $i$ move to an alternative viewpoint $\mathrm{m}$.

\section{The Self-Termination of Viewpoint Planning}

By calculating the angle between the $M$ alternative viewpoints of the viewpoint $i+1$ and all the viewpoints before $i$, if all the angles is less than the threshold of angle $T_{\theta}$, then the viewpoint planning is considered to self-termination. In this study, the threshold of angle $T_{\theta}$ is set to 30 degrees.

\section{EXPERIMENTS AND RESULTS}

The algorithm has been implemented on an automation work cell (see Fig.7 (a)), which composed of a structured light system, two axis turntable, and control panel. The system is equipped with a two axis turntable to enable two degree of freedom.

In this study, the most representative objects are rebuilt to make the results more reliable. Fig.7 (b), (c), (d) shows the 3$\mathrm{D}$ models in our test objects, respectively.

According to the above process, three objects have been reconstructed with viewpoints planning, corresponding viewpoint planned path shown in Fig.8 The viewpoint planning method is consistent with the idea of artificial search object: by obtaining the point cloud of the first viewpoint, rotate the object is to obtain the non-visual area of an object until the completion of the object is measured.

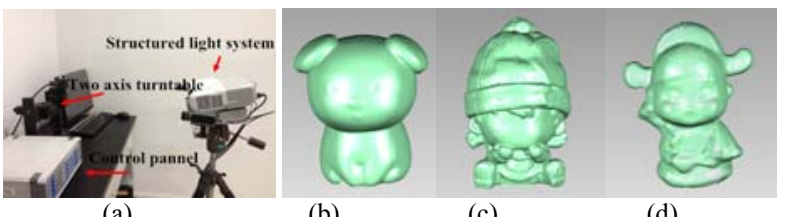

(a)

(b)

(c)

(d)

FIGURE VII. (a) THE AUTOMATION WORK CELL; (b)A TOY DOG MODEL; (c) A GIRL MODEL; (D) A GOD OF WEALTH MODEL
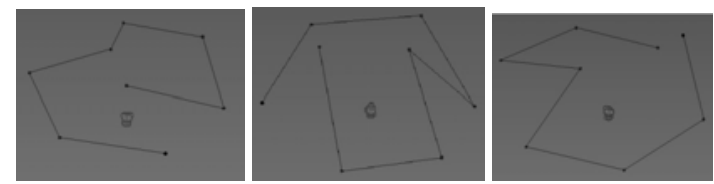

(a) Three cases of a toy dog model

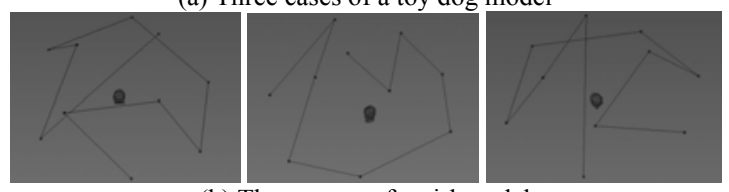

(b) Three cases of a girl model

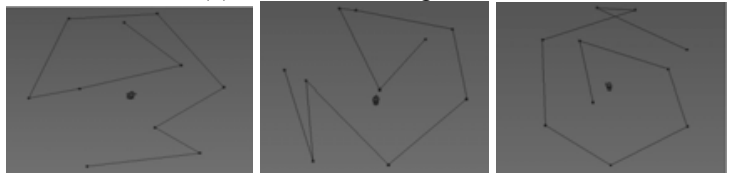

(c)Three cases of a god of wealth model

FIGURE VIII. THE PATH OF VIEWPOINT PLANNING

\section{CONCLUSIONS AND FUTURE WORKS}

In this study, a new digital strategy has been proposed. First of all, the advantage of this method is that the data can be automatically processed, classified, view planned. In short, it can automatically measure the 3-D information based on the structured light system for unknown objects. First, obtained data are analyzed, filtered and classified into WV and BV areas. Second, the NBV is based on the angle criterion and shielding criterion, the rough registration of two point clouds completed by the coordinate transformation, and then the refined registration completed by ICP. Moreover, the BV patches are gathered for identifying potential views, and geometric and physical limitation are taken into account that it computes a set of NBVs in each iteration to provide several positioning scheme of the acquisition system. Future work will consist of improving the degree of freedom of the 
platform, digitizing large objects and in open environment for automatically measuring unknown objects. At the same time, it will introduce mechanism that can evaluate the next viewpoint.

\section{ACKNOWLEDGMENT}

The work was supported by the National Natural Science Foundation of China (Project No. 51175087, 61473090), Fujian Province Outstanding Youth Foundation, (Project No. 2013J06013) and Overseas Studies Scholarship of CSC.

\section{REFERENCES}

[1] Goesele M, Snavely N, and Curless B, "Multi-view stereo for community photo collections," Computer Vision, vol.11, 2007, pp.1-8.

[2] Mauro M, Riemenschneider H, and Signoroni A, "A unified framework for content-aware view selection and planning through view importance," Proceedings of the British Machine Vision Conference, BMVA Press. 2014.

[3] Furukawa, Y, Curless, B, Seitz, "Towards internet-scale multi-view stereo," CVPR, 2010, pp.1434-1441.

[4] Hornung A, Zeng B, and Kobbelt L, "Image selection for improved multi-view stereo," CVPR, 2008, pp.1-8.

[5] Tarbox. G, Gottschlich. S, "Planning for complete sensor coverage in inspection," Computer Vision and Image Understanding, 1995, pp.84111 .

[6] Zha H, Morooka K, Hasegawa T, "Active modeling of 3-D objects: planning on the next best pose (NBP) for acquiring range images,"3D Digital Imaging and Modeling, 1997, pp.68-75.

[7] S. Larsson and J. A. P. Kjellander, "Path planning for laser scanning with an industrial robot," Robot. Auton. Syst, vol. 56, no. 7, 2008, pp. 615-624.

[8] W. R. Scott, "Model-based view planning," Mach. Vis. Appl., vol. 20,no.1, 2009, pp. 47-69.

[9] WHAITE P, FERRIE F, "Autonomous exploration: Driven by uncertainty," IEEE Pattern Analysis and Machine Intelligence, vol.19, no.3, 1997, pp.193-205.

[10] YUAN X, "A mechanism of automatic 3D Object modeling,"IEEE Trans Pattern Analysis and Machine Intelligence, vol.17 no.3, 1995 pp.307-311.

[11] B. Loriot, R. Seulin, and P. Gorria, "Non-model based method for an automation of 3D acquisition and post-processing,"Electronics Letters on Computer Vision and Analysis, 2008, pp.67-82.

[12] B. Loriot, R. Seulin, and P. Gorria, "Non-model based method for an automation of 3D acquisition and post-processing," Electronics Letters on computer, vol.7, 2008, pp.67-82.

[13] Souhaiel Khalfaoui, Ralph Seulin, and Yohan Fougerolle, "An efficient method for fully automatic 3D digitization of unknown objects," Computers in Industry, vol.64, no.9, 2013, pp.1152-1160 